\title{
Alphavirus Vectors for Therapy of Neurological Disorders
}

\section{Kenneth Lundstrom*}

PanTherapeutics, Rue des Remparts 4, CH1095 Lutry, Switzerland

\begin{abstract}
Alphavirus vectors engineered for gene delivery and expression of heterologous proteins have been considered as valuable tools for research on neurological disorders. They possess a highly efficient susceptibility for neuronal cells and can provide extreme levels of heterologous gene expression. However, they generally generate short-term transient expression, which might limit their therapeutic use in many neurological disorders often requiring long-term even life-long presence of therapeutic agents. Recent development in gene silencing applying both RNA interference and microRNA approaches will certainly expand the application range. Moreover, alphaviruses provide interesting models for neurological diseases such as demyelinating and spinal motor diseases.
\end{abstract}

Keywords: Alphaviruses; Gene delivery; Neuronal expression; Gene silencing.

\section{Introduction}

Both viral and non-viral vectors have provided interesting novel approaches in research on neurological disorders with a great potential for future therapeutic applications too [1,2]. The advantages of using non-viral vectors are the simple, rapid and relatively inexpensive vector production although they have suffered from inefficient gene delivery and therefore limited efficacy. Viral vectors have provided excellent delivery and high expression of heterologous genes. On the other hand, production of large quantities of viral vectors is often more complicated and expensive. Furthermore, the application of viral delivery has raised some considerations related to safety issues. Among the nonviral vectors, plasmid DNA complexed with polyethyleneimine (PEI) has been applied for targeting of neuronal stem cells [3]. Moreover, intravenous administration of pegylated immunoliposomes (PIL) carrying plasmid DNA expressing shRNA against the human epidermal growth factor receptor demonstrated $90 \%$ gene silencing in mice [4]. There are a number of viral vectors, which have been evaluated for CNS delivery and even such therapeutic indications as Alzheimer's disease, Parkinson's disease and amyotrophic lateral sclerosis in rodent and primate models [2]. In this context, adeno-associated virus (AAV), herpes simplex virus (HSV), lentivirus and SV-40 virus vectors have been applied. For instance, AAV vectors expressing NGF prevented age-related memory deficit and increased the cholinergic neuron size by $34 \%$ after intraseptal injections [5]. Furthermore, AAV-based NGF therapy in a phase I clinical trial in 8 patients with mild Alzheimer's disease demonstrated no long-term adverse effects, suggested reduced rate of cognitive decline and increase in cortical 18-fluorodeoxyglucose based on PET scans [6]. Similarly, helper-free HSV-1 vectors expressing GDNF and BDNF were evaluated in a rat model for Parkinson's disease [7]. In rats sacrificed 7 months after intrastriatal HSV-1 administration, significantly superior benefits measured as correction of behavioral deficits and protection of nigrostriatal dopaminergic neurons were observed for GDNF in comparison to BDNF. Lentivirus-based GDNF delivery to the striatum and substantia nigra was evaluated in a primate model for Parkinson's disease, in which prevention of loss of dopaminergic neurons and improved motor performance were observed. In aged animals dopaminergic neurons were protected from cell death [8].

Moreover, in vivo delivery of SV40 vectors expressing HIV1 RevM10 was studied in rhesus monkey CNS [9]. Stereotactic injections into the caudate nucleus showed strong neuronal expression throughout the 6 month study. No expression was observed in astrocytes and oligodendroglial cells. SV40-based delivery caused no evidence of inflammation or tissue damage.

Despite these encouraging results obtained with both non-viral and viral vectors described above alternative gene delivery methods are in demand. This review will focus uniquely on alphaviruses, which possess various features related to CNS delivery and make them attractive candidates as gene delivery vehicles. Interestingly, these viruses have a dual function as they are responsible for involvement in certain neurological disorders and simultaneously can be engineered to gene delivery vectors for therapeutic function.

\section{Basics of Alphaviruses}

Alphaviruses belong to the family of Togaviridae and possess a single-stranded RNA of positive polarity surrounded by a capsid and an envelope structure [10]. Several of the members of the alphaviruses are considered as pathogens and have caused epidemics. Recently, a global fever outbreak occurred for Chikungunya [11]. Similarly, Semliki Forest virus (SFV) and Venezuelan equine encephalitis (VEE) virus have been associated with febrile illness in Africa [12] and epidemics in horses and humans in South America [13], respectively. Despite that, SFV [14], Sindbis virus [15] and VEE [16] have been engineered to efficient gene expression vectors, albeit based on attenuated strains. In this context, three major approaches have been taken (Figure 1). (A) Replication-competent expression can be obtained by the addition of a second subgenomic promoter and the gene of interest to the full-length genome. These vectors can provide high levels of heterologous gene expression and simultaneously generate new viral particles. They can sustain longer duration of expression and in vivo re-infect additional

*Corresponding author: Kenneth Lundstrom, PanTherapeutics, Rue des Remparts 4, CH1095 Lutry, Switzerland, Tel: +41 79776 6351; E-mail: lundstromkenneth@googlemail.com

Received November 18, 2011; Accepted December 22, 2011; Published January 02, 2012

Citation: Lundstrom K (2012) Alphavirus Vectors for Therapy of Neurological Disorders. J Stem Cell Res Ther S4:002. doi:10.4172/2157-7633.S4-002

Copyright: ( 2012 Lundstrom K. This is an open-access article distributed under the terms of the Creative Commons Attribution License, which permits unrestricted use, distribution, and reproduction in any medium, provided the original author and source are credited. 
(A) Replication-competent

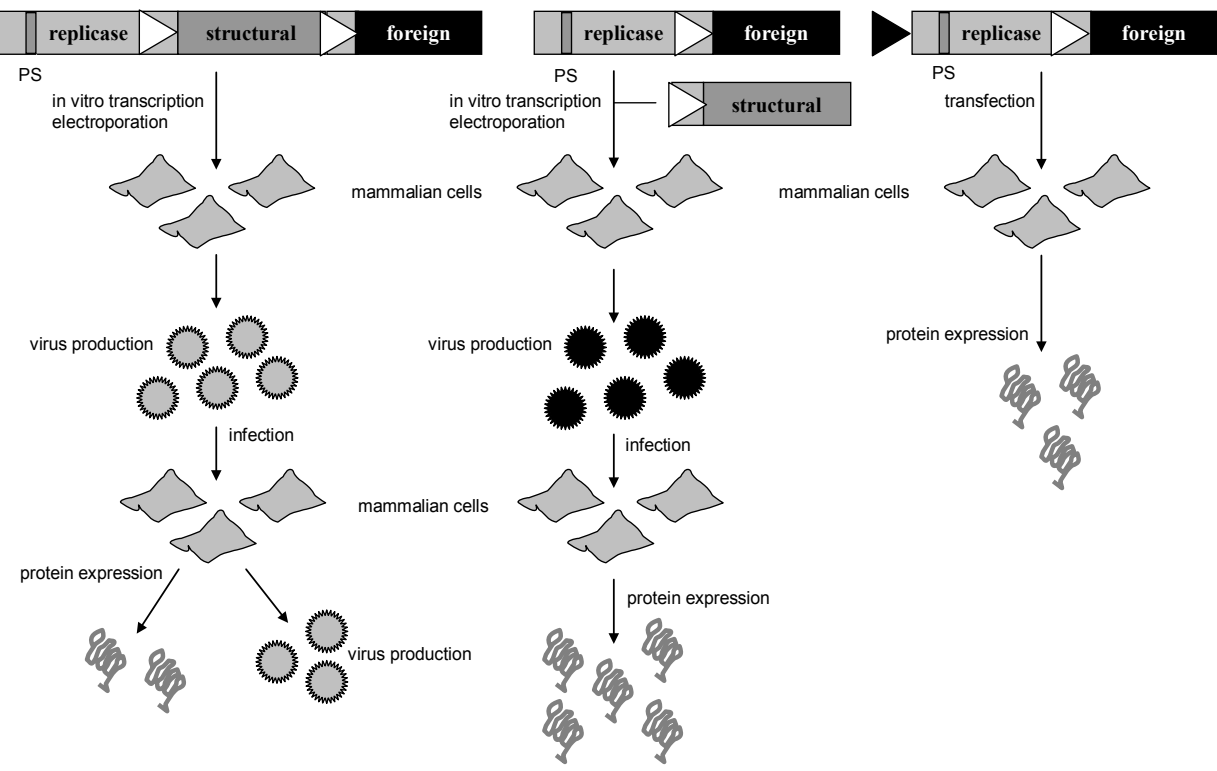

Figure 1: Various types of SFV vectors used for heterologous gene expression. (A) Replication-competent vector: Full-length genome of SFV with an additional SFV $26 S$ subgenomic promoter $(\triangleright)$ upstream of the foreign gene of interest. This vector system generates replication-proficient particles, which after infection produces new progeny and recombinant protein. (B) Replication-deficient vector: In this system, expression and helper vectors are co-electroporated in parallel to generate replication-deficient particles due to the packaging of only RNA carrying the packaging signal (PS). Infection results in recombinant protein expression, but no virus progeny. (C) DNA layered vector: The presence of a CMV promoter $(\checkmark)$ allows direct use of plasmid DNA for cell transfection to generate recombinant protein

cells. However, the downside of this approach is the potential unwanted spread of viral infection. (B) Replication-deficient expression requires the use of a dual vector system consisting of an expression vector including the non-structural replicase genes, a strong subgenomic promoter and the gene of interest. The other vector provides the viral structural genes and is therefore named helper vector. In attempts to generate the highest possible safety of the system, a second generation helper vector was engineered, which rendered the produced viral particles conditionally infectious [17]. Moreover, the design of split helper vectors provided additional safety and guaranteed no production of unwanted wild type recombined virus [18]. Due to the presence of an RNA packaging signal uniquely located on the expression vector, recombinant particles contains only the replicase genes and the gene of interest, which prevents any further production of virus progeny. Replication-deficient vectors can still provide one round of cell transduction and generation of high levels of transient heterologous gene expression. (C) Layered DNA expression is based on alphavirus expression vectors, where the SP6 RNA polymerase promoter has been replaced with a CMV promoter. It allows direct expression of plasmid DNA without any intermediate steps of recombinant particle production. The advantage is obviously the absence of infectious virus, but the necessity of using conventional DNA transfection methods and lack of efficient gene delivery has to be taken into account.

The rapid generation of high-titer ( $10^{9}$ to $10^{10}$ infectious particles/ $\mathrm{mL}$ ) virus particles and the broad host range has made alphavirusbased gene expression suitable for a number of applications. In this context, especially SFV vectors have been used for the expression of topologically different proteins [14,19]. Particularly, the expression of membrane proteins has been very successful indicated by the expression of $\mathrm{G}$ protein-coupled receptors both at high levels [20] and in large numbers [21]. Moreover, they have demonstrated neuronspecific gene expression in primary neurons [22], in hippocampal slice cultures [23] and in vivo in rodent brain [24]. The local high-level expression of $\beta$-galactosidase in the striatum and amygdale of rat brain without any detectable adversity compared to control animals [24] suggested potential use of alphavirus-based delivery for research and even therapy. Additionally, replication-proficient SFV vectors based on the avirulent strain $\mathrm{A} 7(74)$ have been used for gene delivery in primary neurons and in hippocampal slices [25]. Interestingly, the expression was highly glial cell specific at $37^{\circ} \mathrm{C}$, but at $31^{\circ} \mathrm{C}$ the GFP expression was similar to the wild type phenotype demonstrating neuron-specific (up to 94\%) expression. Further development has included using oncolytic SFV vectors against lung cancer [26] and osteosarcoma [27] in mouse models. Recently, intravenous administration of oncolytic SFV-A7 provided therapeutic efficacy in nude mice with human glioma xenografts [28]. Furthermore, in the context of tumor therapy, SFV vectors have been encapsulated in liposomes to provide targeting of tumor tissue [29].

\section{Alphaviruses and Neurological Disorders}

Particularly, the favorable susceptibility of SFV particles in primary neurons in culture [22], in hippocampal cells [23] and in vivo [24] has made these vectors attractive in the research and potential therapy of neurological disorders. Additional encouragement was received from the confirmation of regression of human glioma xenografts in nude mice after intravenous administration [28]. One drawback with applying alphaviruses for therapeutic use is the relatively short duration of gene expression, due to the nature of non-integrating degradable RNA. This refers particularly to replication-deficient vectors. Engineering of replication-competent vectors have provided prolonged expression profiles and allowed re-infection of additional 
adjacent cells. Another issue is the extreme expression level provided from the strong subgenomic alphavirus promoter [10]. To address the above shortcomings, novel vectors have been developed. For instance, mutant SFV vectors with enhanced and prolonged duration of gene expression have been engineered [30,31]. In attempts to reduce expression levels without the loss of gene delivery efficacy, mutations were introduced into the SFV $26 \mathrm{~S}$ subgenomic promoter, which resulted in significantly lower gene expression [32].

In the context of applying alphaviruses for therapy in the CNS, SFV particles expressing EGFP and interleukin-10 (IL-10) were administered intranasally into Balb/c mice [33]. Enhanced IL-10 expression resulted in amelioration of experimental autoimmune encephalitis (EAE) and suggested that the noninvasive intranasal route is potentially successful for protein delivery. In another study on EAE, SFV vectors were employed to express tissue inhibitor of metalloproteinase-2 (TIMP2) and TIMP-3 [34]. Intraperitoneal delivery generated TIMP-2 expression throughout the brain and resulted in the inhibition of EAE development. Although SFV-based TIMP-3 expression resulted in reduced disease it was not statistically significant. These findings may provide the basis for novel treatment of CNS autoimmune disorders such as multiple sclerosis.

Another example of an approach to treat a CNS disorder has been to deliver transforming growth factor beta 1 (TGF-beta1) intraperitoneally by an avirulent SFV vector [35]. Studies in mice with EAE, a model for multiple sclerosis, demonstrated the presence of TGF-beta1 mRNA in the CNS after intraperitoneal administration by in situ hybridization and RT-PCR. A significant reduction in disease severity was observed suggesting a promising approach for treating autoimmune disorders in the CNS. Recently, studies on virus-induced demyelination have served as a model for multiple sclerosis [36]. CNS inflammation and primary demyelination caused by SFV infections was evaluated in wild type and $\gamma \delta \mathrm{T}$ cell knock-out (KO) mice. Although inflammation and demyelination were similar for the two groups the $\gamma \delta \mathrm{T}$ cell $\mathrm{KO}$ animals did not exhibit extensive remyelination even after 35 days post-infection. Immunization of $\gamma \delta \mathrm{T}$ cell $\mathrm{KO}$ mice with an SFV E2 peptide led to elevated antibody production and accelerated remyelination and thereby enhanced recovery and repair of the CNS.

An interesting delivery approach to rat brain, albeit not for treating CNS disorders has been SFV-mediated gene therapy of RG2 rat glioma [37]. Rats with gliomas were treated with SFV particles expressing IL12 via an implanted cannula. Treatment with $5 \times 10 \mathrm{e} 7$ particles showed $70 \%$ reduction in tumor volume, whereas the higher dose of $5 \times 10 \mathrm{e} 8$ particles generated an $87 \%$ reduction. Alphaviruses have also proven their excellent efficacy in CNS delivery after intravenous [28], intranasal [33] and intraperitoneal [34] administration, but their application in treating neurological disorders might to some extent be limited by the transient nature of expression. Therefore, other viruses such as adeno-associated viruses (AAV) and lentiviruses providing long-term expression profiles might be more suitable for these applications [2]. However, new development in gene silencing with the discovery of RNA interference (RNAi) and micro-RNA (miRNA) has provided new possibilities for therapeutic intervention also for neurological disorders [38]. For instance miR-9 and miR-128 are up-regulated in patients with Alzheimer's disease [39,40]. Similarly, other miRNAs have been associated with Parkinson's disease [41], Huntington's disease [42], spinocerebellar ataxia [43] and frontotemporal dementia
[44]. Although there is very little experience of applying alphavirus vectors for RNAi and miRNa delivery the idea is attractive for treating neurological disorders. Mutant and oncolytic vectors with prolonged duration of expression profiles might improve efficacy. Liposome encapsulated SFV particles have demonstrated targeting properties and also protection against recognition by host immune defense systems and might facilitate re-administration of therapeutic agents.

Alphaviruses have to some extent been applied for stem cell research. In this context, lentiviral vectors were targeted to stem cells by incorporation of the membrane-bound human stem cell factor (hSCF) and the Sindbis virus-derived fusogenic molecule (FM) [45]. This approach resulted in efficient targeting of cells expressing CD117, a type III cell surface transmembrane tyrosine kinase receptor, which naturally binds hSCF. In another study, neurotrophic Sindbis virus was verified in a role for survival motor neuron (SMN) protein [46]. It was demonstrated that SMN protected primary neurons and differentiated neuron-like stem cells from virus-induced apoptosis. In contrast, in cultured cell lines no protection was observed.

Interestingly, alphaviruses have been shown to cause encephalitis, deymelinating and spinal motor diseases, which has resulted in extensive research to treat these maladies. For instance the spread of neuroadapted Sindbis virus (NSV) to motor neurons and the spinal cord leads to severe hind limb weakness in mice [47]. It provides a model for human encephalomyelitis in humans. Because naoloxone, a opioid receptor antagonist, has previously been shown to block microglial-mediated neurodegeneration, the drug effect was evaluated after NSV infection. Naloxone treatment prevented paralysis and increased the survival of motor neurons. Due to the targeting of microglial responses similar therapeutic benefit may occur in humans. In another study, mice were subjected to pre-, co- or post-treatment with cationic liposome-DNA complexes (CLDCs) challenged with lethal Western equine encephalitis (WEE) virus [48]. Pre-treatment provided a significant protection and therapeutic effect was obtained up to $12 \mathrm{~h}$ after WEE challenge. CLDC treatment significantly increased IFN-gamma, TNF-alpha and IL-12 levels in the serum. This suggested that strong non-specific activation of innate immunity is capable of eliciting protective immunity against lethal alphavirus strains and providing prophylactic protection.

Aerosol exposure of macaques to VEE was assayed for changes in global gene expression [49]. Major histocompatibility complex (MHC) class I transcripts were induced in the brain and lungs, whereas the expression of S100b, a factor associated with brain injury, was downregulated. Not surprisingly, cytokine-mediated signals and caspases were also affected. This approach provides useful information for host cell responses after aerosol alphaviruses exposure, which will be valuable in understanding and treating CNS disorders.

\section{Conclusions}

Alphavirus vectors have demonstrated excellent capacity of generating extreme levels of heterologous gene expression in a broad range of host cells both in vitro and in vivo. Furthermore, efficient delivery after intravenous, intraperitoneal and intranasal administration can provide highly efficient delivery to the CNS. These features make alphavirus attractive candidates for therapeutic interventions of neurological disorders. SFV vectors have been evaluated in studies on demyelinating disease, a model for multiple 
sclerosis. Furthermore, the promising gene silencing achieved by delivery of RNAi and miRNA sequences could present an interesting approach for the treatment of CNS disorders in the future. Research on the involvement of alphaviruses in certain CNS orders will further improve the understanding of possibilities in both prophylactic and therapeutic intervention.

\section{References}

1. de Lima MC, da Cruz MT, Cardaso AL, Simoes S, de Almeida LP (2005) Liposomal and viral vectors for gene therapy of the central nervous system. Curr Drug Target CNS Neurol Disord 4: 453-465

2. Lundstrom K (2007) Prospects of treating neurological disorders by gene therapy. Curr Opin Investig Drugs 8: 34-40.

3. Falk A, Holmstrom N, Carlen M, Cassidy R, Lundberg C, et al. (2002) Gene delivery to adult stem cells. Exp Cell Res 279: 34-39.

4. Partridge WM (2004) Intravenous, non-viral RNAi gene therapy of brain cancer. Exp Opin Biol Ther 4: 1103-1113.

5. Klein RL, Hirko AC, Meyers CA, Grimes JR, Muzyczka N, et al. (2000) NGF gene transfer to intrinsic basal forebrain neurons increases cholinergic cell size and protects from age-related, spatial memory deficits in middle-age rats. Brain Res 875: 144-151.

6. Tuszynski MH, Thal L, Pay M, Salmon DP, U HS, et al. (2005) A phase 1 clinical trial of nerve growth factor gene therapy for Alzheimer disease. Nat Med 11: 551-555.

7. Sun $M$, Kong L, Wang X, Lu XG, Gao Q, et al. (2005) Comparison of the capability of GDNF, BDNF, or both, to protect nigrostriatal neurons in a rat model of Parkinson's disease. Brain Res 1052: 119-129.

8. Kordower JH, Emborg ME, Bloch J, Ma SY, Chu Y, et al. (2000) Neurodegeneration prevented by lentiviral vector delivery of GDNF in primate models of Parkinson's disease. Science 290: 767-773.

9. Louboutin JP, Marusich E, Fisher-Perkins J, Dufour JP, Bunnell BA, et al (2011) Gene transfer to the rhesus monkey brain using SV40-derived vectors is durable and safe. Gene Ther 18: 682-691.

10. Strauss JH, Strauss EG (1994) The alphaviruses: gene expression, replication and evolution. Microbial Rev 58: 491-562.

11. Kelvin AA (2011) Outbreak of Chikungunya in the Republic of Congo and the global picture. J Infect Dev Ctries 5: 441-444.

12. Mathiot CC, Grimaud G, Garry P, Bouquety JC, Mada A, et al. (1990) An outbreak of Semliki Forest virus infections in Central African Republic. Am J Trop Med Hyg 42: 386-393.

13. Weaver SC, Salas R, Rico-Hesse R, Ludwig GV, Oberste MS, et al. (1996) Remergence of epidemic Venezuelan equine encephalomyelitis in South America. VEE Study Group. Lancet 348: 436-440.

14. Liljeström $P$, Garoff $H$ (1991) A new generation of animal cell expression vectors based on the Semliki Forest virus replicon. Biotechnology (N Y) 9: 1356-1361.

15. Xiong C, Levis R, Shen P, Schlesinger, S, Rice CM, et al. (1989) Sindbis virus: an efficient, broad host range vector for gene expression in animal cells. Science 243: 1188-1191.

16. Davis NL, Brown KW, Smith JF, Johnston RE (1989) In vitro synthesis of infectious Venezuelan equine encephalitis virus RNA from a cDNA clone: analysis of a viable deletion mutant. Virol 171: 189-204

17. Berglund P, Sjöberg M, Garoff H, Atkins GJ, Sheahan BJ, et al. (1993) Semliki Forest virus expression system: production of conditionally infectious recombinant particles. Biotechnology (N Y) 11: 916-920.

18. Smerdou C, Liljeström P (1999) Two-helper RNA system for production of recombinant Semliki Forest virus particles. J Virol 73: 1092-1098.

19. Lundstrom K (2003) Semliki Forest virus vectors for rapid and high-level expression of integral membrane proteins. Biochim Biophys Acta 1610: 90-96.
20. Sen S, Jaakola VP, Heimo H, Engström M, Lundstrom K, et al. (2003) Functional Expression and Direct Visualization of the human $\alpha_{2 B}$-Adrenergic Receptor and a2B-AR - Green Fluorescent Fusion Protein in Mammalian Cell Lines using Semliki Forest Virus Vectors, Prot Expr Purif 32: 265-275.

21. Hassaine G, Wagner R, Kempf J, Cherouati N, Hassaine, et al. (2006) Semlik Forest Virus vectors for overexpression of $101 \mathrm{G}$ Protein-coupled receptors in mammalian host cells. Prot Expr Purif 45: 343-351.

22. Lundstrom K, Schweitzer C, Rotmann D, Hermann D, Schneider EM, et al. (2001) Semliki Forest virus vectors: efficient vehicles for in vitro and in vivo gene delivery. FEBS Lett 504: 99-103.

23. Ehrengruber MU, Lundstrom K, Schweitzer C, Heuss C, Schlesinger S, et al (1999) Recombinant Semliki Forest virus and Sindbis virus infect efficiently neurons in hippocampal slice cultures. Proc Natl Acad Sci U S A 96: 7041 7046.

24. Lundstrom K, Richards JG, Pink JR, Jenck F (1999) Efficient in vivo expression of a reporter gene in rat brain after injection of recombinant replication-deficient Semliki Forest virus. Gene Ther Mol Biol 3: 15-23.

25. Ehrengruber MU, Renggli M, Raineteau O, Hennou S, Vähä-Koskela MJ, et al. (2003) Semliki Forest virus A7(74) transduces hippocampal neurons and glial cells in a temperature-dependent dual manner. J Neurovirol 9: 16-28.

26. Määttä AM, Mäkinen K, Ketola A, Liimatainen T, Yongabi FN, et al. (2008) Replication competent Semliki Forest virus prolongs survival in experimental lung cancer. Int J Cancer 123: 1704-1711.

27. Ketola A, Hinkkanen A, Yongabi F, Furu P, Määttä AM, et al. (2008) Oncolytic Semliki forest virus vector as a novel candidate against unresectable osteosarcoma. Cancer Res 68: 8342-8350.

28. Heikkilä JE, Vähä-Koskela MJ, Ruotsalainen JJ, Martikainen MW, Stanford MM, et al. (2010) Intravenously administered alphavirus vector VA7 eradicates orthotopic human glioma xenografts in nude mice. PLoS One 5: e8603.

29. Lundstrom K (2004) Gene therapy applications of viral vectors. Technol Cancer Res Treatment 5: 467-477.

30. Lundstrom K, Rotmann D, Hermann D, Schneider EM, Ehrengruber MU (2001) Novel mutant Semliki Forest virus vectors: gene expression and localization studies in neuronal cells. Histochem Cell Biol 115: 83-91.

31. Lundstrom K, Abenavoli A, Margaroli A, Ehrengruber MU (2003) Novel Semlik Forest virus vectors with reduced cytotoxicity and temperature-sensitivity for long-term enhancement of transgene expression. Mol Ther 7: 202-209.

32. Lundstrom K, Ziltener P, Hermann D, Schweitzer C, Richards GJ, et al. (2001) Improved Semliki Forest virus vectors for receptor research and gene therapy. J Rec Signal Transd Res 21: 55-70.

33. Jerusalmi A, Morris-Downes MM, Sheahan BJ, Atkins GJ (2003) Effect of intranasal administration of Semliki Forest virus recombinant particles expressing reporter and cytokine genes on the progression of experimental autoimmune encephalomyelitis. Mol Ther 8: 886-894.

34. Nygårdas PT, Grönberg SA, Heikkilä J, Joronen K, Sorsa T, et al. (2004) Treatment of experimental autoimmune encephalomyelitis with a neurotropic alphavirus vector expressing tissue inhibitor of metalloproteinase-2. Scand $J$ Immunol 60: 372-381.

35. Vähä-Koskela MJ, Kuusinen TI, Holmlund-Hampf JC, Furu PT, Heikkilä JE, et al. (2007) Semliki Forest virus vectors expressing transforming growth factor beta inhibit experimental autoimmune encephalomyelitis in Balb/c mice. Biochem Biophys Res Commun 355: 776-781

36. Safavi F, Feliberti JP, Raine CS, Mokhtarian F (2011) Role of $y \delta ~ T$ cells in antibody production and recovery from SFV demyelinating disease. Neuroimmunol 235: 18-26.

37. Roche FP, Sheahan BJ, O'Mara SM, Atkins GJ (2010) Semliki Forest virusmediated gene therapy of the RG2 rat glioma. Neuropathol Appl Neurobiol 36: 648-660.

38. Lundstrom K (2011) Micro-RNA in disease and gene therapy. Curr Drug Discov Technol 8: 76-86.

39. Barbato C, Ruberti F, Cogoni C (2009) Searching for MIND: MicroRNAs in neurodegenerative disease. J Biomed Biotechnol 2009: 871313. 
Citation: Lundstrom K (2012) Alphavirus Vectors for Therapy of Neurological Disorders. J Stem Cell Res Ther S4:002. doi:10.4172/2157-7633.S4002

Page 5 of 5

40. Lukiw WJ (2007) Micro-RNA specification in fetal, adult and Alzheimer's disease hippocampus. Neuro Report 18: 297-300.

41. Kim J, Inoue K, Ishii J, Vanti WB, Voronov SV, et al. (2007) A MicroRNA feedback circuit in midbrain dopamine neurons. Science 317: 1220-1224.317: 1220-1224.

42. Johnson R, Zuccato C, Belyaev ND, Guest DJ, Cattaneo E, et al. (2008) A microRNA-based gene dysregulation pathway in Huntington's disease. Neurobiol Dis 29: 438-445.

43. Lee Y, Samaco RC, Gatchel JR, Thaller C, Orr HT, et al. (2008) miR-19, miR101 and miR-130 co-regulate ATXN1 levels to potentially modulate SCA1 pathogenesis. Nat Neurosci 11: 1137-1139.

44. Rademakers R, Eriksen JL, Baker M, Robinson T, Ahmed Z, et al. (2008) Common variation in the miR-659 binding site of GRN is a major risk factor for TDP43-positive frontotemporal dementia. Hum Mol Genet 17: 3631-3642.

45. Froelich S, Ziegler L, Stroup K, Wang P (2009) Targeted gene delivery to
CD117-expressing cells with lentiviral vectors co-displaying stem cell factor and a fusogenic molecule. Biotechnol Bioeng 104: 206-215.

46. Kerr DA, Nery JP, Traystman RJ, Chau BN, Hardwick JM (2000) Survival motor neuron protein modulates neuron-specific apoptosis. Proc Natl Acad Sci USA 97: 13312-13317.

47. Prow NA, Irani DN (2007) The opioid receptor antagonist, naloxone, protects spinal motor neurons in a murine model of alphavirus encephalomyelitis. Exp Neurol 205: 461-470.

48. Logue CH, Phillips AT, Mossel EC, Ledermann JP, Welte T, et al. (2010) Treatment with cationic liposome-DNA complexes (CLDCs) protects mice from lethal Western equine encephalitis virus (WEEV) challenge. Antiviral Res 87: 195-203.

49. Koterski J, Twenhafel N, Porter A, Reed DS, Martino-Catt S, et al. (2007) Gene expression profiling of nonhuman primates exposed to aerosolized Venezuelan equine encephalitis virus. FEMS Immunol Med Microbiol 51: 462-472.

This article was originally published in a special issue, Cell Therapy for neurological disorders handled by Editor(s). Dr. Pranela Rameshwar, UMDNJ-New Jersey Medical School, USA 